\title{
275 Autoimmune bullöse Dermatosen
}

\section{T. Bieber, A. Steen}

Die erworbenen bullösen Krankheiten im Kindesalter sind relativ selten und klinisch in der Regel sehr schwer voneinander zu unterscheiden. Die diagnostische Klärung erfolgt in der Regel über die direkte Immunfluoreszenz oder das sog. Immunomapping und/oder Immunoblotting.

\section{Juveniles bullöses Pemphigoid}

Das bei Kindern sehr seltene bullöse Pemphigoid ist durch das Auftreten relativ großer und praller Blasen an den Streckseiten der Extremitäten und bei Kindern insbesondere an den Händen und Füßen charakterisiert. Die direkte Immunfluoreszenz zeigt eine lineare Ablagerung von IgG und C3 an der Basalmembranzone. Das Antigen ist ein 180- und/oder 230-kDBestandteil der Basalmembran. Wie bei Erwachsenen erfolgt die Therapie in der Regel über systemische Steroide.

\section{Epidermolysis bullosa acquisita}

Die Epidermolysis bullosa acquisita (EBA) tritt bei Kindern häufiger auf als bei Erwachsenen. Klinisch ist sie nur sehr schwer von einem bullösen Pemphigoid zu unterscheiden. Das Immunomapping und der Immunoblot zeigen eine Reaktivität mit Typ-VII-Kollagen. Vom Verlauf her ist diese Krankheit mit dem bullösen Pemphigoid vergleichbar. Sie spricht ebenfalls auf systemische Steroide an.

\section{Chronisch-bullöse Dermatose der Kindheit und lineare IgA-Dermatose}

Obwohl ursprünglich als getrennte Entitäten beschrieben, stellen die chronisch-bullöse Dermatose der Kindheit (CBDK) und die lineare IgA-Dermatose (LAD) sehr wahrscheinlich nur Varianten ein und derselben autoimmunblasenbildenden Krankheit mit einem 285-kD-Antigen dar. Die klinischen Symptome sind mit denen des bullösen Pemphigoids vergleichbar.

\section{Dermatitis herpetiformis}

Die juvenile Form der Dermatitis herpetiformis (DuhringKrankheit) ist durch das Auftreten von stark juckenden, papulovesikulösen Hautveränderungen bei Kleinkindern charakterisiert. An die mögliche Assoziation mit einer Zöliakie sollte stets gedacht werden. Die Krankheit korreliert ebenfalls mit den Haplotypen HLA-B8 und -DR3. Sie spricht ebenfalls sehr gut auf eine glutenfreie Diät sowie auf Dapson an.

\section{Herpes gestationis des Neugeborenen}

Der Herpes gestationis ist eine autoimmune bullöse Krankheit, die im 2. oder 3. Trimenon der Schwangerschaft auftreten kann. Obwohl die Schwangerschaft und das Kind durch die Krankheit nicht gefährdet sind, kann es zu Frühgeburten kommen, wobei die Kinder gelegentlich ähnliche bullöse Hautveränderungen aufweisen wie die Mutter. Diese werden sehr wahrscheinlich durch den passiven Transfer der Autoantikörper der Mutter, die gegen 180-kD-Antigene der Basalmembran gerichtet sind, ausgelöst.

\section{Literatur \\ - Kap. 280.}

\section{Genodermatosen}

\section{T. Bieber, A. Steen}

In diesem hochkomplexen Bereich der pädiatrischen Dermatologie wurden durch die genetische Forschung in den letzten Jahren erhebliche Fortschritte im Verständnis der Pathophysiologie erzielt. Bei den Genodermatosen handelt es sich in der Regel um monogene Krankheiten, bei denen funktionell relevante Mutationen zur Generierung von abnormen Strukturproteinen führen.

\section{Hereditäre Epidermolysen}

Definition. Die heterogene Gruppe der blasenbildenden Genodermatosen umfasst mindestens 15 Typen, wobei die Charakterisierung der Mutation in Zukunft möglicherweise das Zusammenlegen bzw. Abspalten weiterer Subtypen ermöglichen wird.

Klassifikation und Pathogenese. Auf der Grundlage der klinischen, morphologischen und ultrastrukturellen Befunde können die hereditären Epidermolysen in 3 Gruppen eingeteilt werden.

Bei den intraepidermalen Formen mit einer Spaltbildung oberhalb der Basalmembran konnten Mutationen in den Genen von Keratin 1 und 10 nachgewiesen werden. Bei der besonderen Ichthyosis bullosa Siemens wurden Mutationen im Keratin-2e-Gen festgestellt.

Bei den junktionalen Epidermolysen findet die Spaltbildung in der Basalmembran auf der Ebene der Lamina lucida statt. Hier wurden Mutationen in den Genen von Lamininketten im Gen der $\alpha 6 / \beta 4$-Integrine und im Gen von Kollagen XVII nachgewiesen.

Bei den dermolytischen dystrophischen Epidermolysen findet die Spaltbildung unterhalb der Basalmembran in der papillären Dermis statt. Hier konnten mehrere Mutationen in den Genen vom Kollagentyp VII festgestellt werden. 